\title{
Acute Myocardial Infarction after Dog Bite: A Case Report
}

\author{
A Rahman, A Khan, SM Hasnayeen, AKMM Islam \\ Department of Cardiology, NICVD
}

Key words:
Myocardial
infarction,
Dog bite,
Capnocytophaga
canimorsus.

\begin{abstract}
Acute myocardial infarction (AMI) after dog bite is rare. Capnocytophaga canimorsus, a Gramnegative, commensal organism of canine buccal cavity may cause bacterimia and sepsis. Hypotensioninduced stasis, septic emboli to the coronary artery, endothelial dysfunction and plaque vulnerability induced by acute inflammation may lead to acute coronary syndrome in this situation. Isolation of the bacteria may be challenging due to fastidious nature of the organism. Management is as per standard protocol. However, appropriate antibiotic therapy is crucial. Like many other parts of the world, dog bite is endemic in Bangladesh. The case presented here reminds us of the need for preparedness to deal with myocardial infarction associated with dog bite effectively.
\end{abstract}

(Cardiovasc. j. 2009; 1(2) : 231-233)

\section{Case History}

Mr. MU, a 40-year-old normotensive, non-diabetic but dyslipidaemic smoker was admitted into the NICVD with central compressive chest pain and sweating for 3 hours. He had history of dog bite in the lower limb 5 days back. On examination, he was found apprehended, his pulse was 80 beats per minute, blood pressure $130 / 85 \mathrm{~mm}$ of $\mathrm{Hg}$, temperature $98^{\circ} \mathrm{F}$, and respiratory rate was $18 / \mathrm{min}$. The apex beat was in the left $5^{\text {th }}$ intercostal space, no left parasternal heave, palpable $\mathrm{P}_{2}$ and thrill. The first heart sound $\left(\mathrm{S}_{1}\right)$ was rather soft, without any added sound. Trachea was normal position. Breath sound was vesicular with no added sounds. Examination of other systems revealed normal findings. He received ciprcfloxacin, tetanus toxoid and anti-rabies vaccine, the schedule of the later was completed after admission into the NICVD.

The ECG of the patient revealed ST segment elevation in leads $V_{1}-V_{6}$, I and aVL (Figure 1a) and resolution of ST segment after thrombolytic therapy (Figure 1b). CK-MB was 95 units/L, cardiac troponin I was positive $(17 \mathrm{ng} / \mathrm{ml})$. Echo revealed mild anterolateral hypokinesia with left ventricular EF 40\% (Figure 2a \& 2b). Blood biochemistry including blood sugar, serum creatinine and serum electrolytes were normal. He was diagnosed as a case of acute anterior myocardial infarction with history of dog bite. The patient was managed as per standard protocol with inhalational oxygen, aspirin, clopidogrel, metoprolol and oral nitrate. Streptokinase 1.5 MU was infused for thrombolysis. Further investigation revealed normal total and differential leucocyte counts, hemoglobin and ESR. Microbiological
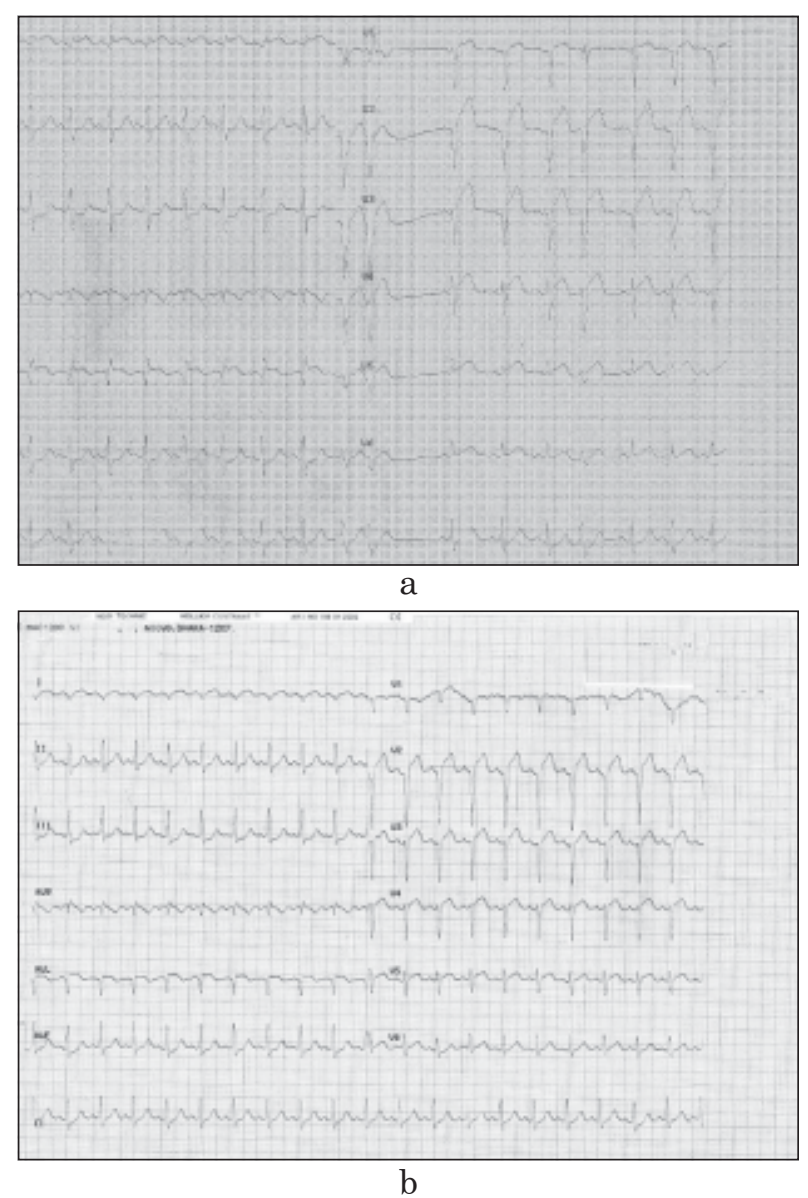

Fig.-1. a. ECG of the patient a. before thrombolytic therapy b. after thrombolytic therapy.

examination did not show any microorganism in the peripheral blood film, also blood culture was negative. Coronary angiography, 5 days after admission, demonstrated insignificant lesion in the 


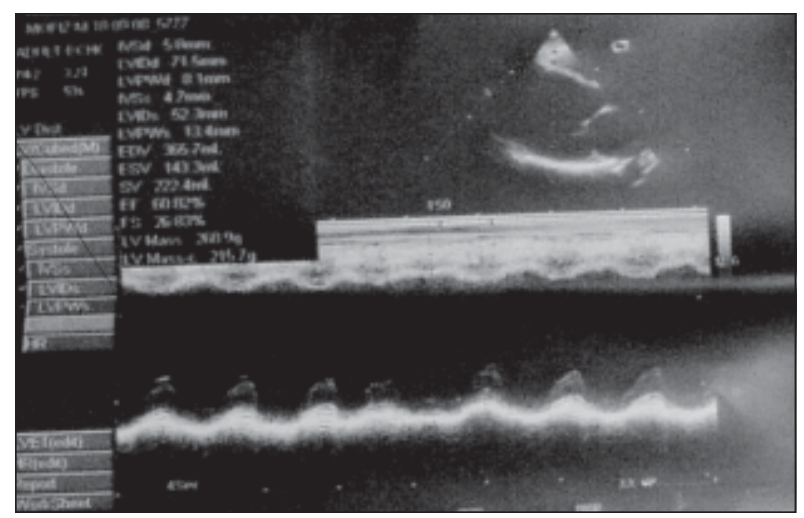

a

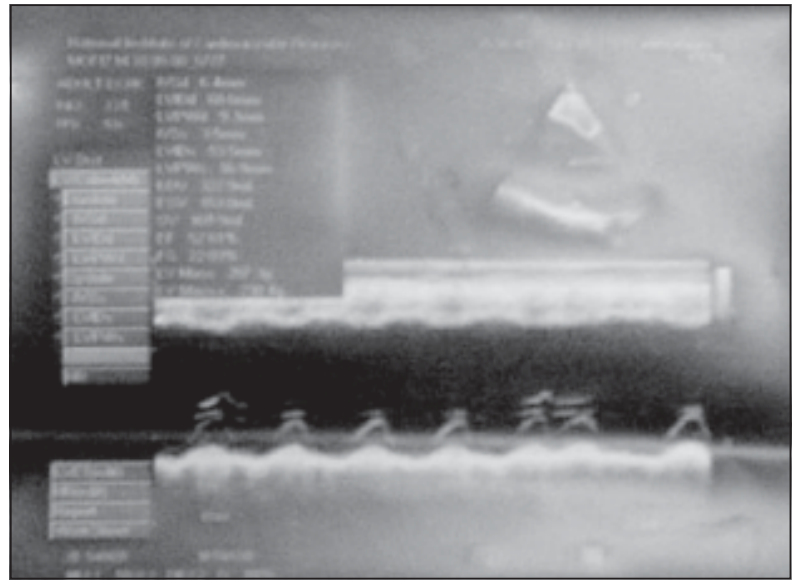

$\mathrm{b}$

Fig.-2a \&b: Echocardiography of the patient showing left ventricular anterior wall hypokinesia.

ramus, otherwise normal epicardial coronaries (Figure 3a \& 3b).

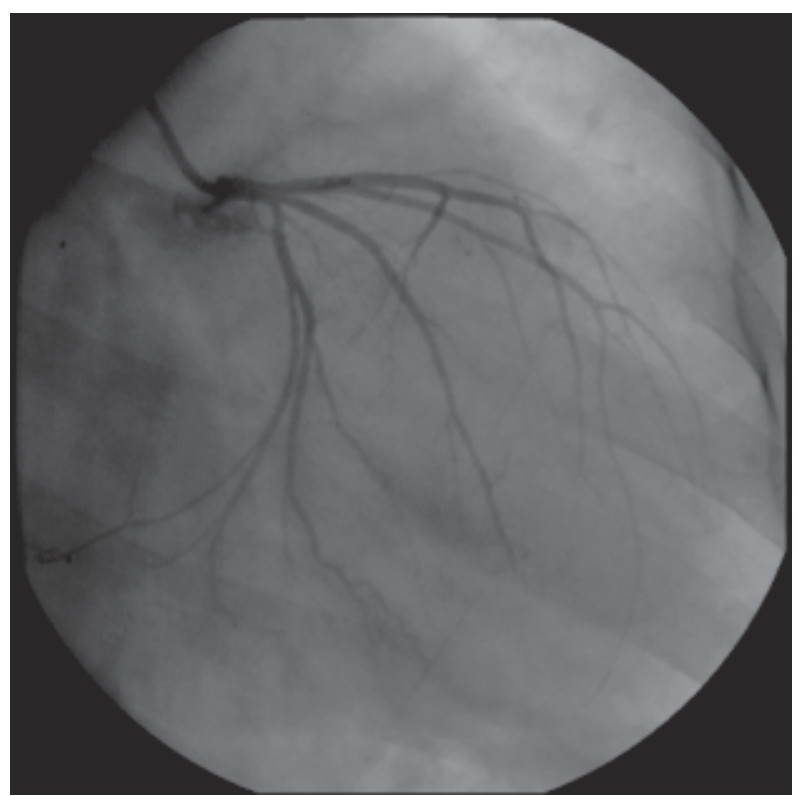

Fig.-3 (a) : Coronary angiogram of the patient showing normal left coronary system.

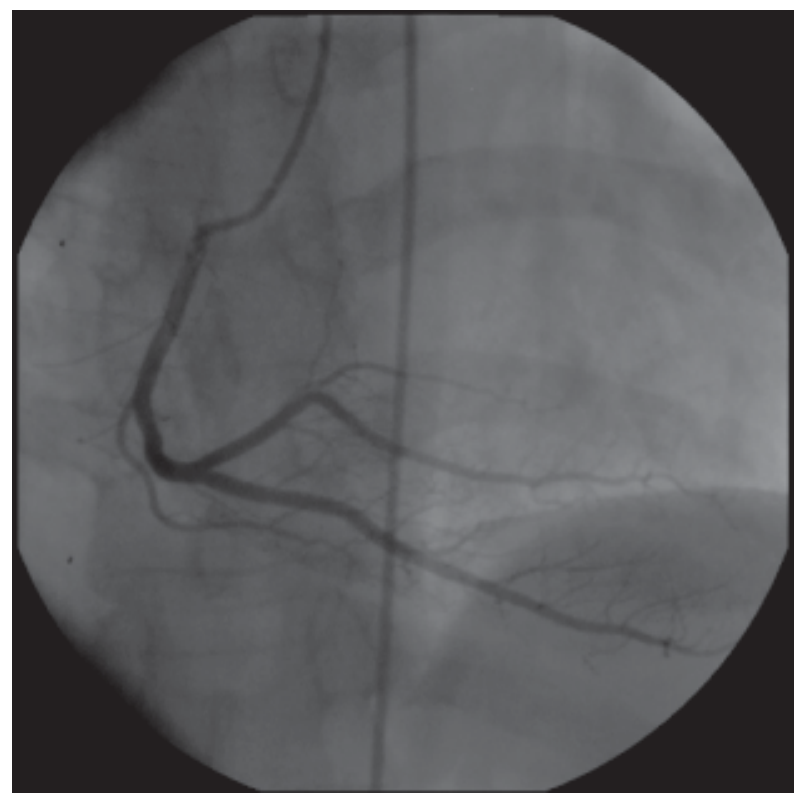

$\mathrm{b}$

Fig.-3 (b) : Coronary angiogram of the patient showing normal right coronary system.

\section{Discussion}

Dog bite has been associated with acute myocardial infarction (AMI). ${ }^{1,2,3} \mathrm{~A}$ number of commensal organisms are known to reside within the buccal cavity of dog, Capnocytophaga canimorsus is one of them. C. canimorsus, formerly known as dysgonic fermenter 2 (DF 2), is a fastidious, Gramnegative rod. ${ }^{4}$ It causes fulminant sepsis following dog and cat bites, particularly in asplenic patients and alcoholics. ${ }^{5}$ Infection and bacteremia may precipitate an $\mathrm{AMI}^{1}$, which usually results from hypotension or from endocarditis with septic emboli to the coronary arteries ${ }^{2}$. Alternatively, inflammation may lead to elevation of acute inflammatory proteins like CRP and amyloid A, as well as, to activation of monocytes and adhesion molecules. All these factors may lead to endothelial dysfunction and make the atherosclerotic plaque more vulnerable to acute coronary syndrome. ${ }^{6,7,8}$ C. canimorsus sometimes cause fulminant myopericarditis which may mimic AMI. ${ }^{9}$ In case of $C$. canimorsus infection, blood counts are usually indicative of acute inflammation, Gram-negative rods may be found in PBF and blood culture may yield specific organism. In our case, signs of acute inflammation were almost absent and no organism was found in PBF and blood culture. This may be due to antibiotic therapy after dog bite. $C$. canimorsus has been reported to be susceptible to 
penicillin, third-generation cephalosporins, quinolones, doxycycline, erythromycin, rifampicin, vancomycin and imipenem in vitro. ${ }^{10}$ Besides this, blood culture in case of $C$. canimorsus infection may be missed or incorrectly identified unless specific laboratory methods are followed e.g. prolonged subculture in $\mathrm{CO}_{2}$ incubation. ${ }^{11}$

Another possibility is that the patient might actually presented with acute myopericarditis, rather than acute myocardial infarction. Acute myopericarditis can cause chest pain, ST elevation in ECG and elevation of cardiac biomarkers including CK-MB and troponin I. ${ }^{9,11,12}$ Presence of near-normal epicardial coronary arteries in coronary angiography was an expected findings in that case. However, rapid regression of STelevation after streptokinase infusion favours the diagnosis of MI.

Dog bite is an endemic in many parts of the world including Bangladesh. It may be a rare cause of AMI. The clinicians should have appropriate knowledge and preparedness to deal with such an uncommon condition.

\section{Financial Disclosure: None.}

\section{References}

1. Scharf C \& Widmer U. Myocardial infarction after dog bite. Circulation. 2000;102:713-714.

2. Ehrbar HU, Gubler J, Harbarth S \& Hirschel B. Capnocytophaga canimorsus sepsis complicated by myocardial infarction in two patients with normal coronary arteries. Clin Infect Dis 1996;23:335-336.
3. Newton NL \& Sharma B. Acute myocardial infarction associated with DF-2 bacteremia after a dog bite. Am J Med Sci 1986;291:352-354.

4. Westwell AJ., Spencer MB \& Kerr KG. DF-2 bacteremia following cat bites. Am J Med 1987; 83:1170.

5. Lion C, Escande F \& Burdin JC. Capnocytophaga canimorsus infections in human: review of the literature and cases report. Eur J Epidemiol 1996;12:521-533.

6. Liuzzo G, Biasucci LM, Gallimore JR, et al. The prognostic value of $\mathrm{C}$-reactive protein and serum amyloid A protein in severe unstable angina. $N$ Engl $J$ Med. 1994;331:417- 424 .

7. Mazzone A, De Servi S, Ricevuti G, et al. Increased expression of neutrophil and monocyte adhesion molecules in unstable coronary artery disease. Circulation. 1993;88:358 -363.

8. Meier CR, Jick SS, Derby LE, et al. Acute respiratorytract infections and risk of first-time acute myocardial infarction. Lancet. 1998;351: 1467-1471.

9. Auer J, Berent R \& Eber B. Myocardial infarction after dog bite. Circulation. 2001;103:e95.

10. Gill VJ. Capnocytophaga. In: Mandell GL, Bennett JE \& Dolin R, editors. Mandell, Douglas and Bennett's Principles and Practice of Infectious Diseases. Philadelphia, PA: Churchill Livingstone;2000. pp. 24412444.

11. Mitchell I, McNeillis N, Bowden FJ \& Nikolic G. Electrocardiographic myocardial infarction pattern in overwhelming post-splenectomy sepsis due to Capnocytophaga canimorsus. Intern Med J. 2002;32:415418.

12. Goldberger AL, Goldberger E. Miscellaneous ECG patterns: pericarditis and pericardial effusion; myocarditis. In: Goldberger AL, Goldberger E, eds. Clinical Electrocardiography. 5th ed. St Louis: Mosby Year Book; 1994:131-133. 